\title{
Predicting Resilience via Social Support and Illness Perceptions Among Patients Undergoing Hemodialysis
}

\author{
Reihane Hajmohammadi, ${ }^{1}$ and Mahmoud Shirazi ${ }^{2,}$ \\ ${ }^{1}$ M.A Student in General Psychology, University of Sistan and Baluchestan, Zahedan, IR Iran \\ ${ }^{2}$ Associate Professor, Department of Psychology, Faculty of Psychology and Educational Sciences, University of Sistan and Baluchestan, Zahedan, IR Iran \\ "Corresponding author: Mahmoud Shirazi, Department of Psychology, Faculty of Psychology and Educational Sciences, University of Sistan and Baluchestan, Zahedan, IR Iran. \\ Tel: +98-9153404219, E-mail: mshirazi@edpsy.usb.ac.ir
}

Received 2017 June 01; Revised 2017 June 22; Accepted 2017 July 10.

\begin{abstract}
Background and Objectives: Chronic renal disease is a threatening condition for the health, economic, and social status of the affected person and his/her family. Patients undergoing hemodialysis encounter mental and health problems; the current study aimed at predicting resilience via social support and illness perceptions among patients undergoing hemodialysis.

Methods: The current descriptive-correlational study had a statistical population including 308 patients undergoing hemodialysis in Kerman, Iran, in 2017. Based on the Krejcie-Morgan table, the minimum required sample size was 169. The sample was selected using a convenience sampling method. Data collection tools were the Connor-Davidson resilience scale, the medical outcome study (MOS) social support survey developed by Sherbourne and Stewart, and the brief illness perception questionnaire developed by Broadbent et al. Data were analyzed using a Pearson correlation coefficient and a stepwise regression analysis via SPSS version 19.

Results: Results indicated that resilience was significantly and positively related to social support $(\mathrm{r}=0.318, \mathrm{P}<0.05)$ and illness perceptions $(\mathrm{r}=0.165, \mathrm{P}<0.05)$. Among the subscales of social support, emotional support, tangible support, and social interaction could predict resilience, and among the subscales of illness perceptions, only cognitive representation could predict resilience. Conclusions: The obtained results demonstrated that resilience was significantly and positively related to social support and illness perceptions. Additionally, the subscales of social support and illness perceptions could predict resilience among the patients undergoing hemodialysis.
\end{abstract}

Keywords: Disease, Resilience, Social Support, Renal Dialysis

\section{Background}

Chronic renal failure is considered as a public health issue worldwide (1). This failure, also called end-stage renal disease, is a permanent condition that requires renal replacement therapy (peritoneal dialysis, hemodialysis, and transplantation) to sustain life (2). Hemodialysis is the most common method of kidney replacement treatment in Iran (3). Although dialysis can increase the lifespan of a patient, it cannot alter the natural course of a renal disease and fully replace the renal function; as a result, patients experience numerous complications and problems (4). The prevalence of chronic diseases is significantly rising worldwide. This condition leads to many deaths in Iran (5). Nearly 1,580,000 patients undergo hemodialysis treatments worldwide (6). According to the available statistics in Iran, about 1200 to 1400 people annually develop renal failure. In 2013, the head of the department of transplantation and special diseases of the Iranian ministry of health mentioned that 32,686 patients with renal diseases were diagnosed in Iran. According to the Iranian ministry of health, over the next 5 years, the number of patients un- dergoing dialysis doubles in the country (7).

A psychological approach widely used among patients with this failure is based on regulating behaviors. This approach suggests that when dealing with a disease or a life-threatening factor, a patient creates overall impressions and certain beliefs about that disease or factor and its treatment in his/her mind. These beliefs are known as illness perceptions (8). This term refers to organized cognitive representations or beliefs that patients have about various characteristics of their disease (9). Regarding a self-regulation model, a number of studies examined illness perceptions (10). Based on this self-regulation model, cognitive and emotional representations face a number of threats caused by a disease. The common sense selfregulation model is a dynamic model that the 2 sets of representations are constantly evaluated and may change at various stages of a disease. The fundamental premise of this model is that people actively solve problems, which aid them to understand the threats to their health (11).

Resilience is one of the factors that affects people's lives and has attracted many researchers' attention over the past decade. Werner was among the first scientists who ap- 
plied the term resilience in the 1970s. Resilience indicates a person's ability to keep his/her mental-biological balance in perilous situations (12). Resilience is a dynamic process of human adaptation to face adverse events and risk factors (poverty, violence, abuse, family issues, illness, and the like) (13).

Another variable associated with stress and resilience that attracted a lot of attention is the social context affecting resilience known as social support (14). Social support received from family and friends plays a key role in dealing with advanced stages of chronic renal failure (15). Social support is a social network providing psychological resources to cope with stressful life issues (16). Several researchers defined social support as the level of affection, companionship, care, respect, attention, and help received by an individual from other people including family members, friends, and the like. Some researchers consider social support as a social reality and others believe that social support arises from people's perceptions (17). Many studies assessed the role of social support in predicting the course of chronic diseases (18). Szeto et al. revealed that social support, as an independent factor, affected survival in patients undergoing hemodialysis (19). In a study aimed at examining the status of social support among patients undergoing hemodialysis in Zanjan, Iran, the results demonstrated that patients receiving desirable levels of emotional, informational, instrumental, and social support, compared with other patients, recovered faster and better (20). Other studies conducted by Asghari, Mohamadi, Falahi Khoshknam, and Tamadon aimed at determining the understanding of the patients with chronic renal failure from advocacy resources in adjustment with hemodialysis revealed that patients received various types of support from different advocacy resources in adjustment with hemodialysis. Moreover, the patients participating in the current study noted that their families provided most of the support they had received (21). Moreover, a study conducted to evaluate the relationship between hardiness and illness perceptions among patients with cancer indicated that hardiness did not play a significant role in the personal control of illness perceptions (22). Another study that examined 81 patients for a long period (6 years) demonstrated that patients had good perceptions of the chronic course of the diseases. Additionally, their perceptions of their diseases considerably changed over a long follow-up period and they grew optimistic views of their own illness perceptions (23). Babaei et al. (24) in a study conducted on 719 patients with diabetes aimed at examining their awareness and understanding of the disease and its complications indicated that the patients had low levels of knowledge about diabetes and its complications and most of them needed training. A longitudinal study
(25) analyzed data on 32,332 patients undergoing dialysis in 12 nations and concluded that low social support and other social and mental factors were significantly associated with a high risk of mortality. Since hemodialysis is one of the most discussed issues related to healthcare worldwide, including Iran (26), carrying out new research to examine the role of social support, illness perceptions, and resilience in managing this disease and reducing its complications is of significant importance. Although many studies are conducted to investigate the effects of social support on various aspects of health and resilience in patients with chronic diseases, few studies are carried out on illness perceptions and almost no studies are performed to evaluate the roles of social support and illness perceptions in predicting resilience among patients undergoing hemodialysis. Lack of research conducted to predict resilience via illness perceptions and social support among patients undergoing hemodialysis indicates the necessity and importance of carrying out the current study.

\section{Objectives}

Since very few studies are conducted to assess the predictive roles of social support and illness perceptions in resilience among patients undergoing hemodialysis, the current study aimed at determining the roles of social support and illness perceptions in predicting resilience among patients undergoing hemodialysis.

\section{Methods}

\subsection{Research Environment and Patients}

The current descriptive study was conducted by the correlational design. The statistical population included 308 patients undergoing hemodialysis in Kerman, Iran, in 2017. The following parameters $(\mathrm{n}=380, \mathrm{P}=0.05, \mathrm{q}=5 \%$, $\mathrm{z}=1.96$, and $\mathrm{d}=5 \%$ ) were used in the Cochran sample size formula and the required sample size was considered 171 subjects. However, with regard to the possibility that some of the patients may not fill out questionnaires completely, a total of 180 questionnaires were distributed among the patients. Finally, 169 completely filled out questionnaires were analyzed. The sample was selected using the convenience sampling method. Using the Krejcie-Morgan table, the minimum required sample size was considered 169 subjects. The inclusion criteria of the study were being 15 years or older, not dealing with any chronic or acute physical-psychological diseases (determined by a psychologist), not abusing any drugs, and being fully interested to participate in the study. The exclusion criteria were all conditions that prevented the continuation of the study (e g, 
physical and mental crises). None of the subjects were excluded from the current study.

\subsection{Questionnaires}

To collect data, 3 questionnaires on illness perceptions, social support, and resilience were used.

\subsubsection{The Illness Perception Questionnaire}

This questionnaire includes 9 items and was developed by Broadbent et al. in 2006. All the items (except for the item examining casual representation) are scored based on a 10-option Likert scale. Each item assesses a subscale of illness perceptions. Five items evaluate cognitive representation including consequences, timeline, personal control, treatment control, and identity. Two items assess emotional representation including concern about the illness and disease generated emotions. One item measures illness comprehensibility. The causal representation is examined by an open-ended response item, which asks a subject to list the 3 most important causal factors in his/her illness. Since examining the cause of the disease was not among the objectives of the current study, this item was removed. In the current study, the cutoff points of this questionnaire were $0-44,45-49$, and $50-80$, which respectively showed low, moderate, and high illness perceptions among the study subjects. According to the studies conducted in 2001, Cronbach's alpha coefficients of the subscales of this questionnaire varied from 0.79 to 0.89 (8). In a study conducted by Soleimani et al., Cronbach's alpha coefficient of the whole scale was 0.87 (27). In the current study, Cronbach's alpha coefficient of the questionnaire was 0.67 .

\subsubsection{The Social Support Scale}

This scale was developed in 1991 by Sherbourne and Stewart. It assesses the level of perceived social support by a subject. It includes 19 items and 5 subscales. The subscales are as follows: tangible support, emotional support, informational support, affectionate support, and positive social interaction. This is a self-report scale scored based on a 5 -point Likert scale, ranging from $1=$ never to $5=$ =always. The lowest score is 19 and the highest score 95. In the current study, cutoff points of this questionnaire were $0-67$, $68-75$, and $76-125$ which respectively showed low, moderate, and high social support among the study subjects. Using a Cronbach's alpha coefficient, the reliability of the scale and its subscales were reported from 0.74 to 0.93 . In a study carried out by Haririan et al. Cronbach's alpha coefficient of the scale was 0.88 (20). In the current study, Cronbach's alpha coefficient of the scale was 0.95.

\subsubsection{The Resilience Scale}

This scale includes 25 items and was developed by Connor and Davidson in 2003 to measure the ability to cope with pressure and threats. Each item is scored based on a 5-point Likert scale ranging from 0 (totally false) to 5 (totally true). In the current study, cutoff points of the questionnaire were 0 - 73, $74-78$, and 79 - 95, which respectively showed low, moderate, and high resilience among the study subjects. Reliability of the scale was 0.93 , which was completely consistent with the results reported by Connor and Davidson. In a study carried out by Hosseini et al. Cronbach's alpha coefficient of the scale was 0.89 (28). In the current study, Cronbach's alpha coefficient of the scale was 0.68

\subsection{Data Collection Method}

Within 2 months, from January to March 2017, the questionnaires were distributed among 169 patients undergoing hemodialysis willing to participate in the current study. These subjects were asked to aid the authors to conduct the study through filling out the questionnaires precisely, honestly, and anonymously. Their gender and age were the only items mentioned in the questionnaires. The subjects were assured of the confidentiality of the collected data.

\subsection{Ethical Consideration}

Initially, as soon as the study proposal was accepted and registered (code number 21421), by referring to hospitals in Kerman, and explaining the main objectives of the study and the method of filling out the questionnaires, the informed consents were obtained from all the subjects and the questionnaires were distributed among them. Whenever a question seemed vague, some additional explanations were also provided. It should be noted that these explanations were provided to avoid any kinds of ambiguity and/or bias. Information related to each subject was not detectable and only a code was assigned to each patient.

\subsection{Statistical Analyses}

The obtained data were analyzed via SPSS version19 using both descriptive (the mean and standard deviation) and inferential (the Pearson correlation coefficient and regression analysis) statistics.

\section{Results}

Demographic information of the subjects is presented in Table 1. In the current study, 169 patients undergoing hemodialysis within the age range of 12 to 77 years were studied; $40.3 \%$ of the subjects were in the age range of 56 
to 66 years; $59.8 \%$ of the subjects were male and $40.2 \%$ of them were female. Moreover, $89.9 \%$ of the subjects were married.

Table 1. The Demographic Information of the Study Subjects

\begin{tabular}{l|c|c}
\hline Variable & & No. $(\%)$ \\
\hline \multirow{2}{*}{ Age, $y$} & $12-22$ & $3(1.8)$ \\
\cline { 2 - 3 } & $23-33$ & $10(5.9)$ \\
\cline { 2 - 3 } & $34-44$ & $15(8.9)$ \\
\cline { 2 - 3 } & $45-55$ & $36(21.3)$ \\
\cline { 2 - 3 } & $56-66$ & $68(40.2)$ \\
\cline { 2 - 3 } Gender & $67-77$ & $37(21.9)$ \\
\hline \multirow{2}{*}{ Marital status } & Female & $68(40.2)$ \\
\cline { 2 - 3 } & Male & $101(59.8)$ \\
\hline
\end{tabular}

Descriptive results (means and standard deviations) related to the variables and their subscales are presented in Table 2.

Table 2. The Means and Standard Deviations of Illness Perceptions, Social Support, and Resilience ${ }^{\mathrm{a}}$

\begin{tabular}{l|c|c}
\hline \multirow{4}{*}{ Variable } & Subscales & $\mathbf{M} \pm \mathbf{S D}$ \\
\hline \multirow{4}{*}{ Illness perceptions } & Total illness perceptions & $51.95 \pm 9.55$ \\
\cline { 2 - 3 } & Cognitive representation & $33.89 \pm 6.84$ \\
\cline { 2 - 3 } & Emotional representation & $9.47 \pm 4.69$ \\
\cline { 2 - 3 } & Illness comprehensibility & $8.57 \pm 1.50$ \\
\hline \multirow{3}{*}{ Social support } & Total social support & $71.02 \pm 8.67$ \\
\cline { 2 - 3 } & Tangible support & $16.92 \pm 1.77$ \\
\cline { 2 - 3 } & Emotional support & $14.02 \pm 2.14$ \\
\cline { 2 - 3 } & Informational support & $15.06 \pm 1.99$ \\
\cline { 2 - 3 } & Affectionate support & $10.07 \pm 1.85$ \\
\cline { 2 - 3 } & Social interaction & $15.22 \pm 1.99$ \\
\hline \multirow{2}{*}{ Resilience } & Total resilience & $75.029 \pm 11.943$ \\
\hline a N=169. & &
\end{tabular}

The descriptive results (the means and the standard deviations) presented in Table 2 showed that the mean of illness perceptions was 51.95 and the means of its subscales, i e, cognitive representation, emotional representation, and illness comprehensibility were 33.89, 9.47, and 8.75 , respectively. Moreover, the mean of social support was 71.02 and the means of its subscales; i e, tangible support, emotional support, informational support, affection- ate support, and social interaction were 16.92, 14.02, 15.06, 10.07 , and 15.22, respectively. Additionally, the mean of resilience was 75.029. Among the subscales of illness perceptions, cognitive representation had the highest mean. Furthermore, among the subscales of social support, tangible support had the highest mean.

Are there any significant relationships among resilience, social support, and illness perceptions?

To answer this question, the Pearson correlation coefficient was used; results of which are presented in Table 3. This correlation test is applied to examine the relationship between at least 2 variables, which are interval or relative. As can be observed in Table 3, resilience was significantly and positively correlated with social support $(\mathrm{r}=0.318, \mathrm{P}=$ $0.000<0.05)$ and illness perceptions $(\mathrm{r}=0.165, \mathrm{P}=0.032<$ $0.05)$. Moreover, social support was significantly and positively related to illness perceptions $(\mathrm{r}=0.163, \mathrm{P}=0.034<$ 0.05).

Table 3. The Results of the Pearson Correlation Coefficients Among Resilience, Social Support, and Illness Perceptions

\begin{tabular}{lccc}
\hline Variables & $\begin{array}{c}\text { Illness } \\
\text { Perceptions }\end{array}$ & Social Support & Resilience \\
\hline $\begin{array}{l}\text { Illness } \\
\text { perceptions }\end{array}$ & 1 & & \\
Social support & $0.163^{\mathrm{a}}$ & 1 & 1 \\
\hline Resilience & $0.165^{\mathrm{a}}$ & $0.318^{\mathrm{b}}$ & \\
\hline $\begin{array}{l}\mathrm{a} P<0.05 . \\
\mathrm{b} P<0.01 .\end{array}$ & & & \\
\end{tabular}

Can the subscales of social support predict resilience among the patients undergoing hemodialysis?

To answer this question, the stepwise regression analysis was applied. This type of regression analysis is used to examine the impacts of several independent variables, which are interval or relative, on a dependent variable. The results presented in Table 4 indicated that among the subscales of social support, i e, tangible support, emotional support, informational support, and positive social interaction, only the emotional support, tangible support, and social interaction met the criteria for the equation and could determine resilience. In the first step, emotional support was entered into the equation and could determine 0.18 of the variance in resilience. In the second step, emotional support and tangible support were respectively entered into the regression equation and determined 0.24 of the variance in resilience. In the third step, emotional support, tangible support, and social interaction were respectively entered into the regression equation and determined 0.25 of the variance in resilience. The other subscales of social support did not meet the criteria for the 
equation. In other words, the standard beta coefficient showed that one unit variation in resilience changed emotional support by 0.43 in the first model, changed emotional support and tangible support by -0.26 in the second model, and changed emotional support, tangible support, and social interaction by -0.26 in the third model.

Can the subscales of illness perceptions predict resilience among the patients undergoing hemodialysis?

To answer this question, the stepwise regression analysis was applied. The results presented in Table 5 indicated that among the subscales of illness perceptions, i e, cognitive representation, emotional representation, and illness comprehensibility, only cognitive representation met the criteria for the regression equation and could determine resilience among the patients undergoing hemodialysis. The other subscales of illness perceptions did not meet the criteria for the equation; therefore, they were eliminated from the equation. In this regard, cognitive representation was entered into the equation and determined 0.06 of the variance in resilience. In other words, the standard beta coefficient demonstrated that one unit variation in resilience changed cognitive representation by 0.25 .

\section{Discussion}

Due to the increasing prevalence of chronic renal disease, many researches should be conducted to examine factors affecting this disease. Results obtained from such studies can be a great help to patients to improve their conditions and efficiently adapt themselves to the disease. Since, so far, no studies are conducted to predict resilience via social support and illness perceptions among patients undergoing hemodialysis, the current study aimed at determining the predictive roles of social support and illness perceptions in resilience among the patients undergoing hemodialysis. The findings of the current study showed that such predictions were possible. Illness perceptions are personal and perhaps unique experiences derived from both concrete perceptual experiences of illness (e $g$, the experience of symptoms) and abstract sources of knowledge (e g, information from health care professionals such as predialysis education before the start of dialysis, or in the media) (29). Several studies demonstrated that illness perceptions had an effective compliance with treatments and was correlated with the results of various treatments (30). The results of the current study demonstrated that resilience was significantly and positively related to social support and illness perceptions. Moreover, the results revealed that resilience was predicted by illness perceptions and social support that increases in illness perceptions and social support increased resilience. The findings of the current study were consistent with the results of the study by Haririan et al. (27). The results of these studies indicated that social support played a key role in rapid recovery in patients undergoing hemodialysis. Furthermore, these results were in line with the results of a number of studies among which a study by Hasannezhad et al. (31) can be mentioned. The results of their study conducted on patients with multiple sclerosis (MS) showed that psychological hardiness and social support were able to predict adaptability among patients with MS. This is while the results of the current study were not consistent with those of Khakpour and Teymori (22), which indicated that hardiness could not predict illness perceptions, based on personal control of the disease among patients with cancer. To explain these results, it can be noted that the presence of social support and the subscales of emotional support, tangible support, and social interaction play significant roles in promoting resilience among patients undergoing hemodialysis. Patients receiving adequate levels of social support can give meaning to their life issues, mental pressure, physical disabilities, and psychological vulnerabilities and reduce their stress through dealing with these problems effectively. Moreover, social support creates a feeling in patients undergoing hemodialysis that they are not alone and they belong to a group supporting them in the time of trouble. On the other hand, resilience is the ability to successfully deal with stress and hardship (32) and is defined as a source enabling people to easily deal with their issues, resist to stressors, and eliminate mental effects caused by those issues and stressors. People who have higher levels of resilience can successfully adapt themselves to traumatic situations such as having a disease. With regard to illness perceptions, it should be mentioned that when a person's perceptions of physical, emotional, social, and cognitive functions increase, the illness severity, illness course, history of hospitalization, need for surgery, and number of recurrences gradually decline.

\section{Conclusions}

Based on the results of the current study, it can be concluded that social support and illness perceptions played predictive roles in resilience among the patients undergoing hemodialysis. Therefore, the subscales of illness perceptions, as important psychological phenomena and the subscales of social support play key roles in resilience among patients. Patients' perceptions of their disease make them more aware of their physical conditions; hence, this deep understanding of the disease aids patients to attempt to adapt themselves to their disease. In other words, when a patient has a good understanding of his/her health status, his/her resilience increases. Moreover, social 
Table 4. The Results of the Regression Analysis Conducted to Predict Resilience via the Subscales of Social Support

\begin{tabular}{|c|c|c|c|c|c|c|c|}
\hline Steps & Variables & $\mathbf{R}$ & $\mathbf{R}^{2}$ & Adjusted $\mathbf{R}^{2}$ & B & $\mathbf{T}$ & Sig \\
\hline Step 1 & Emotional support & 0.43 & 0.19 & 0.18 & 0.43 & 6.30 & 0.000 \\
\hline \multirow{2}{*}{ Step 2} & Emotional support & \multirow{2}{*}{0.50} & \multirow{2}{*}{0.25} & \multirow{2}{*}{0.24} & 0.53 & 7.44 & 0.000 \\
\hline & Tangible support & & & & -0.26 & -3.67 & 0.000 \\
\hline \multirow{3}{*}{ Step 3} & Emotional support & \multirow{3}{*}{0.52} & \multirow{3}{*}{0.27} & \multirow{3}{*}{0.257} & 0.38 & 3.57 & 0.000 \\
\hline & Tangible support & & & & -0.37 & -4.13 & 0.000 \\
\hline & Social interaction & & & & 0.26 & 0.97 & 0.05 \\
\hline
\end{tabular}

Table 5. The Results of the Regression Analysis Conducted to Predict Resilience via the Subscales of Illness Perceptions

\begin{tabular}{lcccccc}
\hline Variables & R & $\mathbf{R}^{2}$ & Adjusted R2 & B & T & Sig \\
\hline Cognitive representation & 0.25 & 0.06 & 0.06 & 0.25 & 3.45 & 0.001 \\
\hline
\end{tabular}

support aids patients to feel supported and helps them to know that they are supported in the time of trouble.

\subsection{Practical Recommendations}

- Carrying out similar studies on other diseases

- Conducting studies on other factors affecting illness perceptions among patients undergoing hemodialysis and carrying out studies on patients with different cultural backgrounds

- Holding workshops aimed at familiarizing patients undergoing hemodialysis with the disease

- Providing several brochures by cultural, educational, and medical authorities to increase the awareness of patients undergoing hemodialysis

\subsection{Limitations}

Among the limitations of the current study unwillingness of some patients due to their poor physical and mental conditions, and low literacy or illiteracy of some of the subjects (solved through reading the questionnaires to them) can be mentioned.

\section{Acknowledgments}

The current article was derived from a Master's thesis in general psychology approved by University of Sistan and Baluchestan. The authors greatly thank all the patients who helped to conduct the current study.

\section{References}

1. Silva SM, Braido NF, Ottaviani AC, Gesualdo GD, Zazzetta MS, Orlandi Fde S. Social support of adults and elderly with chronic kidney disease on dialysis. Rev Lat Am Enfermagem. 2016;24:e2752. doi: 10.1590/15188345.0411.2752. [PubMed: 27508920].
2. Jansen DL, Grootendorst DC, Rijken M, Heijmans M, Kaptein AA Boeschoten EW, et al. Pre-dialysis patients' perceived autonomy, selfesteem and labor participation: associations with illness perceptions and treatment perceptions. A cross-sectional study. BMC Nephrol. 2010;11:35. doi: 10.1186/1471-2369-11-35. [PubMed: 21138597].

3. Elahi N, Dastom M, Kardaani M. Effectiveness of patient education based on Professional Collaboration of Care Centered Model (PCCC) on self- care in patients on Hemodialysis. Jundishapur J Chron Dis Care. 2014;3(2):17-24.

4. Jafari M, Mannani R, Zarea K. The Association Between Self-Concept and Self-Efficacy in Patients Under Treatment by Hemodialysis. JundishapurJ Chron Dis Care. 2015;4(3) doi:10.5812/jjcdc.27222v2.

5. Rambod M, Rafii F. Perceived Social Support and Quality of Life in Iranian Hemodialysis Patients. J Nurs Scholarship. 2010;42(3):242-9. doi: 10.1111/j.1547-5069.2010.01353.x.

6. Karadag E, Kilic SP, Metin O. Relationship between fatigue and social support in hemodialysis patients. Nurs Health Sci. 2013;15(2):164-71. doi: 10.1111/nhs.12008.

7. Heydari Jame Bozorgi M, Madadizade F, Saber Mahani A. Compare the quality of life for hemodialysis and peritoneal dialysis patients in Kerman. J Soc Med. 2015;1(2):104-9.

8. Valipour F, Rezaiy F. Perception of disease in patients with diabetes and its relation to blood sugar control in patients in Tohid Hospital. Sci J Kurdistan Univ Med Sci. 2012;18:17-9.

9. Al-Smadi AM, Ashour A, Hweidi I, Gharaibeh B, Fitzsimons D. Illness perception in patients with coronary artery disease: A systematic review. Int J Nurs Pract. 2016;22(6):633-48. doi:10.1111/ijn.12494.

10. Hirsch D, Ginat M, Levy S, Benbassat C, Weinstein R, Tsvetov G, et al. Illness perception in patients with differentiated epithelial cell thyroid cancer. Thyroid. 2009;19(5):459-65.

11. Brink EVA, AlsEN PIA, Cliffordson C. Validation of the Revised Illness Perception Questionnaire (IPQ-R) in a sample of persons recovering from myocardial infarction - the Swedish version. Scandinavian Journal of Psychology. 2011;52(6):573-9. doi:10.1111/j.1467-9450.2011.00901.x.

12. Ahmadi R, Sharifi Daramadi P. Evaluate the effectiveness of resilience training on mental health of people with substance dependence alder center in Tehran. J Clin Psychol. 2014;13(2).

13. Grünberg J, Verocay C, Rébori A, Amaral C. The promotion of patient resilience in a pediatric dialysis unit: a case report. Humane Med. 2005;5(2). 
14. Windle G, Bennett KM, Noyes J. A methodological review of resilience measurement scales. Health Qual Life Outcomes. 2011;9(1):8. doi: 10.1186/1477-7525-9-8.

15. Lakzaiy H, Mansouri A, Bamari F, Khamri M, Nori Sancholi H. The Relationship between perceived social support and resiliency in diabetic patients referred to diabetes clinic Ali Asghar Zahedan.J Diabetes Nurs Midwifery Zabul. 2014;2(4):16-24.

16. Rafiei F, Rambod M, Hoseini F. Hemodialysis patients perceived social support. J Nurs Midwifery Tehran Univ Med Sci. 2015;15(1):12-5.

17. Moradi M, Cheraghi A. The relationship between social support and psychological well-being and depression: the mediating role of self-esteem and basic psychological needs. Evol Psychol Psychol Iran. 2015;11(43).

18. Alipour A. Social support associated with immune parameters in healthy subjects, the overall impact model. Think Behav. 2008;12(2):139-4.

19. Szeto CC, Chow KM, Kwan BC, Law MC, Chung KY, Leung CB, et al. The impact of social support on the survival of Chinese peritoneal dialysis patients. Perit Dial Int. 2008;28(3):252-8. [PubMed: 18474917].

20. Haririan H, Aghajanlu A, Ghafori Fard M. Check the status of social support in hemodialysis patients in Zanjan. Med J Islamic Azad Univ. 2013;23(1):80-74.

21. Asghari M, Mohamadi E, Falahi khoshknam M, Tamadon M. Illness perception chronic renal failure patients from advocacy resources in adjustment with hemodialysis: A qualitative study. J Crit Care Nurs. 2010;3(4):142-33.

22. Khakpour M, Teymori S. Relationship between hardiness and illness perception in cancer patients. First International Conference on Psychology and Behavioral Sciences. .

23. Tasmoc A, Hogas S, Covic A. A longitudinal study on illness perceptions in hemodialysis patients: changes over time. Arch Med Sci.
2013;9(5):831-6. doi: 10.5114/aoms.2013.38678. [PubMed: 24273565].

24. Babaiy G, Soltanian A, Khalkhali H, Rabieian M, Bahreini F, Afkhami M. Check the knowledge of people over 18 years Bushehr to identify diabetes and its complications using the general linear models. J Health Payavard. 2015;1(1):52-8.

25. Untas A, Thumma J, Rascle N, Rayner H, Mapes D, Lopes AA, et al. The associations of social support and other psychosocial factors with mortality and quality of life in the dialysis outcomes and practice patterns study. Clin JAm Soc Nephrol. 2011;6(1):142-52. doi:10.2215/CJN.02340310. [PubMed: 20966121].

26. lashkari F, Shariat A, Baraz S, Latif M. Collaborative Care Model effect on the patients' sleep quality with maintenance hemodialysis. Jundishapur J Chron Dis Care. 2013;2(1,2):1-7.

27. Soleimani M, Azimaian S, Yonesi J. The Relationship between perception of illness and coping styles in children of mothers with diabetes. J Diabetes Metabol. 2014;14(3):209-16.

28. Hoseini Almadani A, Ahadi H, Karimi Y, Bahrami H, Maazedain A. Compare resiliency, style, identity, spirituality and social support in addicts, addicts and improved. J Addict Stud. 2012;6(21):47-64.

29. Jia Z, Jayanti A, Foden P, Wearden A, Mitra S. Illness Beliefs in End Stage Renal Disease and Associations with Self-Care Modality Choice. Plos One. 2016;11(7):e0154299. doi: 10.1371/journal.pone.0154299.

30. Nehir S, Tavșanli NG, Ozdemir C, Akyol T. A Determination of Hopelessness and the Perception of Illness in Cancer. OMEGAJ Death Dying. 2017:003022281770433. doi:10.1177/0030222817704336.

31. Hasan Nezhad N, Hodavand Khani F, Ahmadi P, Khanjani Z. The relationship between hardiness and social support, compatibility study on multiple sclerosis. J Med Sci Yazd Martyr. 2013;21(4):74-65.

32. Wu G, Feder A, Cohen H, Kim JJ, Calderon S, Charney DS, et al. Understanding resilience. Front Behav Neurosci. 2013;7 doi: 10.3389/fnbeh.2013.00010. 